\title{
Análisis semántico y hermenéutico de los términos chaka, chakana y chakata ${ }^{1}$
}

\section{Semantic and hermeneutic analysis of the terms chaka, chakana and chakata}

\author{
Claudia Cisneros Ayala \\ Universidad Nacional de Mayor de San Marcos, Lima, Perú \\ ccisnerosa@unmsm.edu.pe \\ https://orcid.org/oooo-0002-2281-2638
}

\begin{abstract}
Resumen
Este artículo estudia la resemantización y determinación del significado de los términos quechua chaka, chakana y chakata. La investigación se centra en la explicación de la estructura morfológica. En el procedimiento partimos de la obra del Lexicón de 1560 donde hallamos el término chakata con significado de cruz. Para realizar el trabajo, se optó por el método filológico-hermenéutico y el uso de diccionarios quechuas. Como resultado del análisis, el término chakata tiene como lexema a chaka que indica el elemento de base que hace cruzar «X» elementos. Chakana sería el objeto con el que se hace cruzar los entes. Finalmente, la palabra chakata, aglutina al sufijo -ta con significado 'inter' o 'entre', siendo la palabra en conjunto chakatay 'entrecruzar'. En conclusión, chakata significaría 'entrecruc-'; en sentido filosófico, forma la base que entrecruza, «X» elementos dentro de la naturaleza y acciones humanas.
\end{abstract}

Palabras clave: semántica, hermenéutica, chaka, chakana y chakata

\begin{abstract}
This paper studies the resemantization and meaning determination of the Quechua terms chaka, chakana and chakata. The research focuses on the explanation of the morphological structure. In the procedure we start from the work of the Lexicon of 1560 where we find the term chakata with the meaning of cross. The philological-hermeneutic method and the use of Quechua dictionaries were chosen for the work. As a result of the analysis, the term chakata has chaka as lexeme, which indicates the base element that makes «X» elements cross. Chakana would be the object with which the entities are crossed. Finally, the word chakata, agglutinates the suffix -ta with meaning 'inter' or 'between', being the word as a whole chakatay 'to cross'. In conclusion, chakata would mean 'inter-cross-'; in a philosophical sense, it forms the basis that intertwines, « $\mathrm{X}$ » elements within nature and human actions.
\end{abstract}

Keywords: semantics, hermeneutics, chaka, chakana, and chakata

Recibido: 15-02-2021

Aprobado: 29-01-2021

Publicado: 15-07-2021

1 Este artículo forma parte de la investigación de la tesis de doctorado de Lingüística que presento en la Universidad Nacional Mayor de San Marcos (UnMSM). 


\section{Introducción}

El presente escrito refiere al tema de la interpretación y resemantización de la semasiología del término chakata y sus variantes, que puede referir a elementos y procesos bases imprescindibles para la naturaleza y el ser humano. El trabajo ha sido encargado por Genaro Quispe Pineda, mi maestro de identidad lingüística y cultural andina, en el año de 1998, para el curso de investigación de pregrado; luego, presentada en la maestría de la Universidad Nacional de Educación Enrique Guzmán y Valle (UNE), los cuales no se concluyeron porque no se contaba con el esquema de investigación cualitativa que se maneja hoy en día; por ello, fue relegándose hasta hoy.

En la etapa de los antecedentes, se consigna dos momentos, el primero desarrolla el contexto histórico que parte desde los diccionarios del siglo XVI y XVII. Los sermonarios, los catecismos que han sido consignados con un pensamiento de origen hispano-latino como narra Ciucci y Muysken (2011) refiriendo a Hernando de Alcocer, en donde se lee: «La ignorancia de la lengua quechua y la consiguiente falta de padres que podían trabajar con los indígenas, era un problema actual [...]» (p. 363). Torres Rubio (1619), en la edición del Catecifmo, cõfefionario y Jermonario en la lengua aymara escribe: "[...] aquí è hecho imprimir pareciēdome,

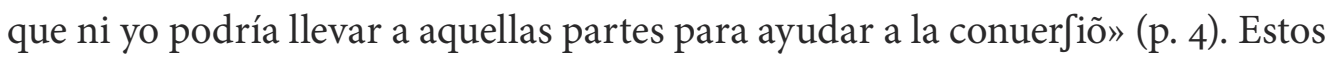
documentos históricos son bases para armar el contenido del artículo. Y como segunda, están las investigaciones contemporáneas que han sido trabajadas por aquellas personas que sienten la necesidad de la reinterpretación histórica y la reivindicación de nuestra historia.

El objetivo general consiste en la interpretación y la resemantización a través del análisis morfosintáctico de su propia estructura morfológica de los códigos chaka, chakana, chakata y chakatasqa. En los objetivos específicos propusimos hallar, sistematizar, razonar, analizar, contextualizar, reinterpretar y precisar la semántica cultural y sus derivaciones de los términos en estudio. El método utilizado fue el método filológico, que interactúa con la hermenéutica y la interdisciplinariedad, aplicado a la bibliografía histórica como un estudio fenomenológico y etnográfico, como propone Parker (1972):

[...] las nuevas líneas del desarrollo de la investigación lingüística, especialmente en el campo de la etnohistoria andina: al confrontar las informaciones lingüísticas con los datos proporcionados por la investigación arqueológica y etnológica, se van multiplicando y enriqueciendo las hipótesis de trabajo. (p. 47)

336 Lengua y Sociedad. Revista de Lingüística Teórica y Aplicada 
El uso de los diccionarios quechuas de diferentes épocas es el soporte del trabajo indicado por Porras Barrenechea como método de investigación. El contexto del estudio la ubicamos en los estudios históricos y filológicos, y a su vez, como «objeto de estudio en sí mismo» (García, 2014, p. 114), porque los términos se extrajeron a partir del Lexicón o Vocabulario de la Lengua General del Perú, llamado Quichua de fray Domingo de Santo Tomás, de 1560, para someter al análisis estructural, ambiental, teórico, lingüístico y conceptual. La técnica investigativa sigue la secuencia de pasos clásicos propuesto por García (2014), la búsqueda de la información (heurístico), la interpretación de los datos en su contexto (hermenéutico), el análisis de la documentación ajustada al contexto, de la descripción del documento, la traducción, la transcripción literal y la crítica del documento, el entorno histórico y lingüístico, la evaluación y el comentario de la muestra en estudio.

Indistintamente también García (2014), citando a García de Cortázar, afirma que «ante la sospecha de imprecisión, ambigüedad o parcialidad del material histórico se debía aplicar con rigor las técnicas de análisis y crítica de los datos» (p. 114); sobre todo, refiriendo a Codoñer, García señala que «consultar [...] diversas ciencias auxiliares de la historia para tomar la decisión de utilizar el método filológico, [...] cuyo fin es el estudio y análisis de los textos, por ende, la filología, [...] "pretende dar una explicación del texto"» (p. 114).

El marco de la investigación se encuentra dentro de las teorías de las ciencias fácticas, documentales e históricas. El tipo, enfoque y diseño de investigación es cualitativa por ser un «enfoque multimetódico» y por acercarse a una interpretación natural del elemento estudiado, en el que el investigador capta al hecho estudiado en el contexto de los fenómenos de base para interpretarlos. Es un trabajo no experimental y descriptivo porque profundiza el análisis interpretativo contextualizando el fenómeno estudiado. No contiene una secuencia lineal, tiene una profundidad de significado y estos se extraen de los mismos datos. Para García (2014), el análisis y el estudio del texto debe abarcar el cómo, el cuándo y de dónde ha llegado el texto, así como también conocer el entorno histórico de la palabra en el que estaba situado el texto y el análisis lingüístico del documento. A ello le suma el análisis gráfico, para llegar a la totalidad de la interpretación textual, además de diversas técnicas de investigación como la descripción, la traducción, la transcripción literal y crítica, el conocimiento del entorno histórico y lingüístico del propio documento, y la evaluación y el comentario lógico de los significados. Los instrumentos obtenidos fueron las fuentes primarias y secundarias de los documentos históricos interdisciplinarios. La muestra refiere 
a la misma palabra chakata, a sus lexemas y morfemas semánticamente tergiversados en su acepción hispana.

La estructura del artículo presenta una introducción que cuenta la contextualización, la evaluación de los marcos teóricos, la plasmación de los objetivos y su justificación de la estructura del escrito. Después, se ubica la revisión bibliográfica histórica y contemporánea sobre chaka, chakana, chakata y chakatasqa para segmentar una base adecuada. Para ello se toman los escritos de Jerónimo de Oré (1598), Gonzales Holguín (1608/1989); Bertonio (1612/2011); Antonio Ricardo (1584); Anónimo (1586/2014) y diccionarios quechuas del siglo XVI y XVII como base de nuestra fundamentación. También trata sobre los estudios contemporáneos de diferentes autores entre ellos Krögel (2020), Parker (1972), entre otros.

En la tercera parte del trabajo, se realiza, por un lado, el análisis e interpretación morfosemántico del término relacionado con un contexto histórico, filosófico y filológico que vincula a chakata como chaka- 'cruce' con un sufijo verbalizador -ta, equivalente a 'entre o inter', como también -cific, -fic. Como categoría lingüística, el término chaka no es sustantivo ni verbo. El sufijo - ta es polifuncional y el sufijo -sqa- funciona como un participio, como un subordinador y/o potencializador. Por otro lado, se realiza una mirada reflexiva y razonada sobre los términos estudiados comparando el uso y la lógica de la naturaleza, en la que llegamos a entender como vínculos de los eventos «de lo que existe». Finalmente, se presentan las conclusiones.

\section{Fuente colonial y contemporáneo sobre los términos chaka, chakana, chakata y chakatasqa}

La teoría entendida como reglas, principios y bases como modelos de ideas que visibiliza a las acciones de los hechos naturales y humanos, y estos codificados y envueltos como ropaje por un idioma quechua; entendido así, se formula como hipótesis que el término chakata asignaría a unos principios vitales de conexión e interconexión de las bases, procesos y hechos de las leyes físicos y químicos existentes en la «Madre Tierra», que son entes que hacen cruzar y entrecruzar lo existente y no solo en una partecita, sino como una totalidad; concepto totalmente muy distinto de lo que consigna en el Lexicón o vocabulario de la lengua general del Perú del siglo Xvi (1560).

338 Lengua y Sociedad. Revista de Lingüística Teórica y Aplicada 


\subsection{Fuente de documentación colonial sobre los términos chaka, chakana y chakata}

La manipulación arbitraria en la utilización de préstamos de los términos hispanos, de palabras completas o acoplando sufijos quechuas a los lexemas hispanos o viceversa en la estructura de las lenguas originarias, como asegura Adelaar (2020), citando a Durston, que el «spanish colonial administrators used specific varieties of Quechua as a lengua general ('general language') and made efforts to artificially standardize it as a unified language» (p. 6). Y como también, sugiere la sexta de passione Domini noftri Iefu Chrifti de Jerónimo de Oré, en la que se concibe la palabra chakata solamente en su traducción hispana y dice: «Christianocuna, Iesu Christop churin: huaquilla cafpa, crusta munaycuffun, Dios yayanchicta hullpuycuspa Adoraffuntac» (Oré, 1598, p. 130). La palabra cruz no se traduce al quechua y solo se acopla sufijos quechuas para formar una sola unidad. Asimismo, se escribe "huchanchic raycum, checan Dios cafpatac cruzpi huañurcan, runa caynillampi. Dios caynimpomca manam huañurqãchu; viñay cauçacmi» (Oré, 1598, p. 130).

En las dos citas anteriores, se aprecia que la palabra cruz se escribe de dos maneras: en la primera cita, se realiza con un fonema /s/ alveolar, anterior, sordo y en el segundo con un sonido $/ \theta /$ fricativo, interdental, sordo. Además, tengamos en cuenta que la acepción hispana etimológica de cruz

viene del latín crux, crucis, en concreto de su acusativo cruce(m). Crux se refería a cualquier instrumento de tortura hecho de palos cruzados, como la horca (de furca, hecha de dos palos) y el tripalium (instrumento de tortura de tres palos). La cruz más famosa, por supuesto, es en la que condenan a Jesús. (deChile.net, s. f. a)

Y según el prólogo a la Breve declaración del Arte y Bocabulario de la lengua del Ynga conforme al estilo y vso de la provincia de Quito, citado en Ciucci y Muysken (2011), alude a:

[...] solo por milagro se aplican al ministerio humilde de predicar a los pobres [indígenas, LC], siendo assi que es de tanta gloria de Dios por el gran fruto y provecho que logran los predicadores de la lengua del Ynga con su predicasion y doctrina, y mas siendo tan facil el predicarles sin la molestia, y tormento, de discurrir agudesas: solo con declararles la palabra evangelica llana y sencillamente. O quiera la bondad divina que nos apliquemos todos con mucho feruor a este misterio, si humilde muy glorioso, para que no se 
malogre en estos miserables Yndios el precio de la sangre y redemción de Jesu Christo. (p. 363)

En la parte de Vocabulario y phraxis de la lengua General, de los indios del Peru, llamada quichua (Anónimo, 1586), se consigna a chaca como: "puente, cadera, anca $[y]$ ronquera» con una misma escritura; luego con palabras aglutinadas con morfemas como «chacacunca. Ronco, chacana. Efcalera de madera o tres eftrellas q llamã las tres marias. chacani atrave $\int a$ ar alguna co $\int a$ en otra, o atracar puerta.

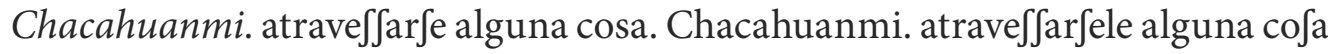
o tener ronquera. Chacayani. enronq̃cer $е$ ». (p. 43). En este Vocabulario y phraxis, la diferencia de escritura no se observa, y tiene la misma forma y el significado de chaka no solamente es cruz, sino tiene otras extensiones que puede nombrar a otros elementos, los cuales hace inferir que puede referirse a eventos que suceden en la naturaleza. Mientras que Gonzáles Holguín (1608/1989) designa a chaca como puente, cadera y ronco:

[...] Puncochaca. Vmbrales o lumbrales. / [...] Chacatullu. Puentezuela huesso de las caderas que las junta. / [...] Chacancani. Estoy ronco. / [...] Chacana. Escalera. / Chacca na. Tres estrellas que llaman las tres marias. / [...] Chhacarayani. Atrauesarse o no caber en alguna cosa angosta. / [...] Chacatascca. Crucificado, o atrauessado vno con otro. / Chacaycusca. Cosa atrauesada cerrado impedido el passo. / Chhacani o quimrayaychani. Atrauesar alguna cosa sola y no dos. / Chacahuanmiconcay. Atrauesarse en la garganta algo, o cerrarse con flemas. / [...] Chhaccayani. Enronquecerse. (pp. $83-84$ )

En el diccionario quechua de 1608, también, se inicia a diferenciar los fonemas entre las consonantes glotalizadas, las aspiradas y las simples; dando a entender que no existe una sola forma de pronunciación; por ende, las traducciones también van diferenciándose, según la pronunciación y escritura, como vemos en la cita precedente. Asimismo, chaka provendría de una palabra aymara, que significa puente para pasar, según Bertonio (1612/2011, p. 322).

\subsection{Fuentes contemporáneas sobre los términos chaka, chakana y chakata}

En los estudios contemporáneos, el estudio de la palabra chakana tiene diferentes acepciones como base de palabra; por eso, Krogel (2021), refiriendo al poema 
«Chakana» de Mariana del Rocio Anchatuña Rojas, asevera que utiliza «el término para conectar entre el cielo y la tierra, como sentido de conexión de dos puntos o como proceso de ascender o descender» (p. 136); en el sentido filosófico se entendería como una trayectoria de interconexión de dos elementos: «wichayankapaq yanapay» 'ayúdame a subir' (p. 136).

Una acotación importante en el análisis de Krogel (2021, p.137) es la diferencia de significado según su escritura entre chakana y chaccana, citando a Gonzáles Holguín, (1608/1989), en donde chakana codifica el significado de 'escalera', y chacca na como 'Tres estrellas que llaman las tres marías' (pp. 83-84). Esta referencia hace distinguir significados. La siguiente cita refiere que chakana es la constelación más famosa del hemisferio Sur: «Sirve además como una brújula, donde las estrellas g crucis y a crucis marcan la dirección al polo sur celeste» (EcuRed, s. f.); en la cual se confirma que tienen como referencia un plano o línea, en la que se une un plano con otro o línea para crear un ángulo de noventa grados o dos rectas que se unen en el mismo plano formando cuatro ángulos rectos y así, formando una cruz invertida (ver Figura 1). Y la secuencia lógica sigue el patrón de unir dos puntos.

Figura 1. La forma de T o cruz invertida de la chakana

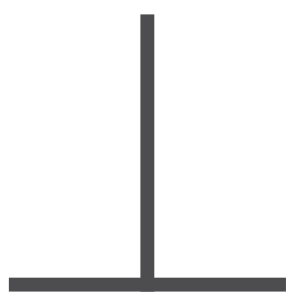

Nota. Elaborado por Clauda Cisneros Ayala.

De la misma manera, también existe una agrupación de estrellas llamada «la cruz del sur [...] y un arbusto cactácea xerofítica nativo del altiplano cuyos frutos se conocen como achakana, que tiene la forma de estrellas agrupadas, que está reproducido en la textilería andina principalmente chompas, llijlla (aguayos), chuspa/istalla [...] y gorras (lluchu)» (Yampara, 2005, p. 32). Asimismo, en la tesis Análisis de las manifestaciones culturales que caracterizan a los cuatro Raymicunas en el Cantón Otavalo y Cotacachi en la provincia de Imbabura, se manifiesta que: 
La Chacana o Cruz significa, puente, transparencia, paso, etc. Está formada por dos líneas:

La línea central representa al hombre, es decir a todo lo masculino en la concepción andina, corresponde también a la creación, al espíritu, a la herencia, a la sucesión. La línea horizontal corresponde a lo creado, la materia, la mama pacha, la permanencia, cuando las dos líneas que marcan los niveles del hombre y la mujer como individuos, si superponen la horizontal sobre la vertical, no al medio exacto sino a la altura del corazón, formando la chakana. El centro [significaría] el vacío, el no conocimiento, lo inimaginable, lo verdadero, lo sagrado [...]. (Moreno, 2012, pp. 25-26)

En otra información hallada sobre la palabra chacana, según Pino (2012), citando a Esterman, alude a puente o cruce, «[...] el símbolo andino de la racionalidad del todo [...] como "puente cósmico"» (p. 45); de igual modo en Timmer (2003), en La Chakana, refiere que «[s]e puede reconocer que un símbolo con cuatro brazos, como el fundamento, el diseño del cosmos, ha estado [sic] conocido por muchas culturas en el mundo» (nota al pie de página n. ${ }^{\circ} 4$ ).

Quevedo (2012) en su tesis denominado, Análisis de contenido del programa La Chakana y sus mensajes de interculturalidad en la radio CCE, citando al De la Torre, afirma que:

la Chakana, de dice que es una cruz con 4 estrellas, un puente hacia el cielo, la fuerza de la tierra y el Sol [...] [y] aparece en los grabados de Chavín, en las estelas de piedra Tiawanako, en los tejidos Wari, en los ceramios Nasca, en los bordados Inka, hoy en día se pueden hallar en las vasijas medicinales. (p. 33; cursivas agregadas)

El Diccionario de la antropología boliviana (Szabó, 2008) remite que el término chakana se origina de dos etimologías aymara y quechua o de una variación de

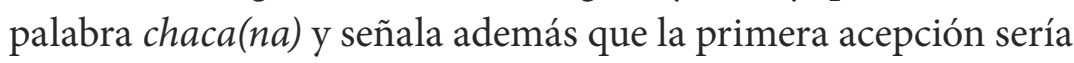

'puente' o escalera. Puede ser una especial como la «escalera de palos atrauesados», igual que el 'kallapu'. En la cosmovisión andina, a nivel simbólico, muchas veces es interpretado como "lo que une las fuerzas"; 2. en la 'astronomía andina' generalmente dan este nombre a la 'Cruz del Sur', sin embargo, Lara lo identifica como la constelación de Libra; [...] 4. denominación de la 'cruz' andina debido a su forma escalonada y rol simbólico. (p. 191) 
De la misma forma, Zúñiga (2006), citando a Milla Villena, sostiene que la chakana en general es la «[...] constelación de la Cruz del Sur o Crux Australis. Ella rige el cosmos andino, ubicando su punto equinoccial en la noche del 2 al 3 de mayo, [...] a partir de esta fecha uno de los ciclos anuales andinos» (pp. 39-40). Finalmente, Del Carpio (2008) en su trabajo «Gestión Pública Intercultural», refuerza que «[...], la Cruz del Sur o chakana, es la [...] aplicación de esta constelación en la distribución territorial de los principales centros ceremoniales, cuyo centro lo ocupa Tiwanaku, a partir de la cual se distribuyen Pukara, Cusco, Uru Uru, Putuxsi» (p. 20).

Es visible que la mayoría concluye en referir a varios elementos y todos afirman que la información refiere a la estrella Cruz del Sur. Partiendo de esta premisa hacemos la inferencia que, en la concepción filosófica, diríamos es algo que une dos extremos de algo, en el caso de la estrella Cruz del Sur, une los dos polos, norte y sur.

\section{Desarrollo del análisis e interpretación de los términos chaka, chakana, chakatasqa y chakata}

\subsection{Análisis de la estructura interna de las palabras en estudio}

Iniciamos con la siguiente reflexión:

Mientras no se realicen estudios de esta índole en un grado exhaustivo, nuestro conocimiento de la gramática quechua se limitará a listas de sufijos con encuestas e informantes notas sobre sus funciones, más relevantes de la naturaleza lexicográfica que gramatical de la lengua y los trabajos de tipo histórico-comparativo y dialectológico no podrán pretender al conocimiento de su estructura profunda. Es muy de lamentar que, hasta la fecha, la madurez insuficiente de la teoría generativa haya dificultado su aplicación a las descripciones gramaticales. (Parker, 1972, p. 46)

Para realizar el estudio se extrajo cinco palabras desde el Lexicón quechua de 1560 como lexema chaka- aglutinado con los sufijos -ta, -sqa, -pi. 
1. chacatapi churani, güi Crucificar

2. chacata Cruz

3. chacatapi churasca Crucificado

4. chacatasca Cosa cruzada

5. Chacatapi churani, güi Crucificar, poner en cruz

(De Santo Tomás, 1560/1951, p. 119)

En la que se evidencia, que tres de ellos, chacatapi churani, güi; chacatapi churasca; chacatapi churana, güi, va unido a un verbo churay para expresar una sola palabra estas palabras estarían construidos con un esquema mental hispana, puesto que culturalmente existen términos únicos para expresar estas palabras en quechua como chakatani, chakatasqa y chakatay. Por ello, elaboramos un contexto histórico, filosófico y filológico de las frases y oraciones extraídas a partir del Lexicón de Fray de Santo Tomás del siglo xvi. En una parte de las annotaciones generales de la lengua aymara en el vocabulario breve de los vocablos, refiere "que ay en efta doctrina la palabra "chacata» [que] significa perderfe y una variante como un infinitivo verbal «chakayata» «perder» (Antonio Ricardo, 1584, p.80).

En el diccionario de 1586, la palabra chaka, chakana, chakatasqa y chakata tiene como lexema base a chaka y con varios significados como chaca/ch'aka/ 'ronquera', chaca/chaka/ 'anca', chaca/chaka/ 'cadera', chaca/chaka/ 'puente' (Anónimo, 1586/2014, p. 79). De la misma manera, la palabra chacata/chakata/ según el Anónimo significa "palo atravesado a manera de cruz. Chakatani / chakata - $y$ / poner en el palo, crucificar. Chacatasqa /chakata-sqa/. Crucificado» p. 79). Para nuestro objetivo, nos quedamos con el concepto de "palo atravesado a manera de cruz». Para justificar ello, se analizó la estructura interna de las frases y oraciones quechuas que indican la objetividad de los significados; luego, se explica la manera en que se habría producido el cambio.

La primera y la quinta frase tienen la misma estructura morfológica. En una primera instancia, como quechuahablante y docente de quechua, se pensó que la oración habría sido un intento de construcción de frase de un hispanohablante, colocando la estructura mental de L1, la lengua hispana, porque para un quechuahablante, solamente, se habría construido como chakatay 'crucificar'; chakatani 'crucifico'. Por esta construcción con dos palabras separadas para construir un verbo 'crucificar', hace pensar que pudo haberse forzado dicha palabra. En lo particular, una característica del quechua, muy obvia, es la de ser bisilábica; por ende, tres sílabas quechuas contarían con una aglutinación de un sufijo, lo cual 
resultaría una modificación en su estructura, siempre manteniendo el significado de la base y un ligero movimiento de giro semántico.

Figura 2. Análisis de la oración chacatapi churani, gui

\begin{tabular}{l|l|l|l|l|l|l}
\hline \multicolumn{3}{l}{ Chacatapi churani, gui } & \multicolumn{3}{l}{ Crucificar / Crucificar, poner en cruz } \\
\hline chaka- & -ta- & -pi & Chura- & -ni & güi & Pues, pongo en la cruz \\
\hline puente & verbalizador & en & pong- & -o & pues & Hago el proceso de puentear \\
\hline
\end{tabular}

Nota. Análisis elaborado por Clauda Cisneros Ayala.

En este sentido, la construcción correcta en una oración actual sería: Runata chakatani 'crucifico al hombre', en donde el primer sufijo - ta, es objeto directo y en la palabra chakatani, el sufijo -ta, sería un verbalizador de nombre. Según Amorín (2007, pp. 101 -102), este sufijo tiene cuatro funciones: 1. El de complemento directo de un verbo transitivo, 2. la de un objeto indirecto, 3. el de indicar una meta que acompaña a un verbo de movimiento con actor humano y respondiendo a las preguntas ¿dónde? y ¿A dónde? 4 . Y como marcador adverbial que va ligado a modificadores verbales constituido por sustantivos temporales, adjetivos, temporales, adjetivos, preadjetivos y numerales de tiempo provenientes del castellano. Y según Cerrón-Palomino (2008) este sufijo - $t a$ funciona como acusativo que «marca al objeto directo o complemento de un verbo transitivo o transitivisado. [...] y responde a las preguntas pi - ta -taq ¿a quién? e imatataq ¿qué?» (p. 121).

Por la construcción analizada podemos afirmar que el sufijo - $t a$ tiene una quinta función: transformar nombres y adjetivos en verbos, con un significado filosófico a manera de entrecruzar con palos atravesados como en el techo de una casa andina o algo similar, puesto que existe términos y oraciones como la siguiente: Chaqllawan chakatay 'entrecruza con palos largos'.

En su estructura interna de la segunda palabra chakata ubicamos a cada uno con su significado literal, lexema chaka 'puente' y un sufijo -ta verbalizador en la que, la traducción no sería 'cruz', sino 'crucific-, entrelaz-, interconect-, entrecruz', sin ningún sufijo de actor verbal o infinitivo verbal, por lo que la palabra tendría como lexema cruc- y el sufijo -ific- o - fic-indicador de acción, además de un sufijo que crea verbos denominales y/o adjetivales. En quechua su equivalente sería los sufijos transformativos - ya y - cha, información inferida, como docente de quechua, a partir de algunos ejemplos hispanos y traducidos al quechua como 
Análisis semántico y hermenéutico de los términos chaka, chakana y chakata Claudia Cisneros Ayala

'clarificar, hacer claro', que en quechua sería achikyachiy; 'fortificar, hacer más fuerte', kallpachay; 'intensificar, hacer intenso', aswanyachiy; ' justificar, hacer lo justo', chiqapchay, 'magnificar, hacer más grande', hatunyachiy; 'osificar, hacerse hueso', tulluchakuy; entre otros ejemplos hispanos encontrados en deChile.net (s. f. a), la traducción a quechua es nuestra.

Figura 3. Análisis en la palabra chakata

\begin{tabular}{l|l|l}
\hline \multicolumn{2}{l|}{ Chacata } & Cruz \\
\hline chaka- & -ta- & Crucific- o entrelaz-, interconect-, entrecuz- \\
\hline puente & Verbalizador de N. & \\
\hline \multicolumn{2}{l}{ Lexema verbal } & Y no tendría sentido de cruz \\
\hline
\end{tabular}

Nota. Análisis elaborado por Claudia Cisneros Ayala

Actualmente, una oración correcta sería chakata apamuy 'trae la escalera', donde el sufijo - $t a$ funciona como un objeto directo, mientras que en runata chakatani 'crucifico al hombre', el sufijo - $t a$ funciona como sufijo verbalizador. Entonces, la traducción sería 'crucific-, enlaz-', o 'entrecruz-' y no cruz como se planteó en el Lexicón de fray Domingo de Santo Tomás (1951). Adicionalmente, a partir de este lexema verbal, se puede flexionar y formar categorías verbales como chakatani 'crucifico', chakatanki 'crucificas', chakatan 'crucifica' y sus plurales. Pero, en el sentido filosófico cultural, estaría dado por entrelazar, entrecruzar, interconectar dos, tres, cuatro, cinco o $\mathrm{N}$ elementos.

Analizando la estructura interna de la tercera frase indicamos que la palabra está formada por dos palabras, para decir un solo significado, el cual se habría formado a partir del nombre chaka sustantivo, convertido en un verbo agregándole el sufijo - $t a$ verbalizador de nombre, con un sentido de 'interconectar'. Luego, se acopló el sufijo - pi circunstancial de lugar o de tiempo preposición hispana con significado 'en' para formar la palabra chakatapi; por tanto, nuestra inferencia sería que se ha forzado la construcción de la frase para poder hacer pensar en la crucifixión humana. Esta frase en la oración actual, no se utiliza, sino un verbo quechua chakatani 'crucifico' o 'entrecruzo'; aquí, ya se entiende que se crucifica en palos atravesados en $\mathrm{N}$ direcciones, cuatro, tres o dos direcciones.

346 Lengua y Sociedad. Revista de Lingüística Teórica y Aplicada 
Análisis semántico y hermenéutico de los términos chaka, chakana y chakata

Claudia Cisneros Ayala

Tabla 4. Análisis de la estructura interna de chacatapi churasqa

\begin{tabular}{l|l|l|l|l|l|l}
\hline \multicolumn{3}{l}{ Chacatapi churasqa } & \multicolumn{2}{l}{ Crucificado } \\
\hline & & & & habí- & -a & \\
\hline chaka- & ta & -pi & Chura- & -sqa & & Había entrecruzado [en palos]. \\
\hline puente -ar & en & Puest- & -o & & Había puesto en el entrecruzado. \\
\hline
\end{tabular}

Nota. Cuadro elaborado por Claudia Cisneros Ayala.

En el análisis de la cuarta y última frase inferimos que el significado real surge a partir del análisis de su estructura interna de la palabra. El término chakatasqa está formado por un lexema base chaka- 'puente, cadera, estrella del cruz del sur' y unido por un sufijo - ta; aquí, el sufijo - ta adquiere como significado de preposición entre o inter, el cual estaría formado de in + ter con significado 'entre' o 'en intervalos, en medio de'. Información parafraseada a partir de las etimologías de la palabra entre que proviene de la voz latina inter, compuesto de in que significa 'hacia dentro, penetración o estar dentro', y de un sufijo -ter, contrastivo (cf. deChile.net, s. f. c).

Desde luego, si se agrega el sufijo -sqa se transforma en un participio del pluscuamperfecto, del pasado narrativo en la que se señala que un verbo indica a un hecho no contado por propia experiencia del informante, sino que ha recibido por parte de una tercera persona, sobre alguna información que ocurrió en unos tiempos inmemoriales no corroborada, o cuando habría tenido dos años el/ la informante sin capacidad de recordar lo realizado. Otra función que podría cumplir el sufijo -sqa es la de convertir al verbo del pasado narrativo en un subordinador. Esto sucede agregándole a un lexema verbal, el sufijo -sqa; luego, los sufijos de referencia de personas para formar una sola unidad.

Figura 5. Análisis de la palabra Chacatasqa

\begin{tabular}{|c|c|c|c|c|c|}
\hline \multicolumn{5}{|c|}{ Chacatasqa } & \multirow[t]{2}{*}{ Cosa cruzada } \\
\hline & & & & Lo que & \\
\hline chaka- & $-\mathrm{ta}$ & & -sqa- & & entrecruzado \\
\hline puentear & verbalizador & en & -ado & \multirow{2}{*}{$\begin{array}{l}\text { un sufijo ausente } \\
\text { de tercera persona } \\
\text { que indicaría un } \\
\text { subordinador. }\end{array}$} & \multirow[b]{2}{*}{$\begin{array}{l}\text { Puesto con los brazos y } \\
\text { piernas estirados en algún } \\
\text { instrumento cruzado. }\end{array}$} \\
\hline cruz- & entre- & & & & \\
\hline
\end{tabular}

Nota. Análisis elaborado por Claudia Cisneros Ayala. 
En relación, al término chakatasqa, también, se consultó a Doña Abelina Ayala Laurente, mi madre, bilingüe, quechua-castellano hablante de Ayacucho, Ocros Cceraocro, de 86 años de edad, el o9 de octubre de 2020, donde corroboró y comentó sobre la siguiente pregunta: ¿Imaynataq chakatasqaqa kanman? ¿Cómo sería o cómo se definiría chakatasqa?'; su respuesta fue con un ejemplo: «Es igual a lo que le hicieron a Túpac Amaru, jalar con cuatro caballos en cuatro direcciones, eso sería chakatasqa», afirmó.

Se volvió a preguntarle ¿Qué más podría ser chakatasqa? En respuesta, comentó no acordarse más palabras relacionadas con la frase en cuestión. Entonces, la deducción sería: sí, Túpac Amaru murió jalado por cuatro caballos y estirados con soga en cuatro direcciones, eso sería verbo chakatay, y no cruz, sino entrecruzar, dándose así, otra idea de la función de - ta como 'entre'.

\subsection{Interpretación lógica de los términos chaka, chakata y chakana}

En el análisis morfosemántico del término chaka, chakana, chakatasqa y chakata, constatamos la acepción literal de sus equivalentes del idioma quechua con el idioma castellano, puesto que una lengua aglutinante tiene la objetividad en su estructura interna, los cuales indican funciones y equivalencias literales para la traducción hispana.

La interpretación lógica se realizó teniendo en cuenta la conexión o vínculo que existe desde un punto A hacia otro punto B, como sugería, el Q’aqi runa, Genaro Quispe Pineda, en una conversación de formación cultural y lingüística. Analizando con esta lógica, el pensamiento quechua codificaría a chaka como «elementos bases», pudiendo ser un lugar, un estado físico, biológico, un tiempo y/o un entendimiento de una persona, que hace extensión respectiva a otra acción de igual proporción, para hacer pasar, cruzar, conectar con otros elementos. Entendido de esta manera, la palabra chakata cobra gran importancia en su asignación de su significado. Entonces, chakata, para la cultura andina, realizaría la conexión e interconexión de vínculos de los eventos del kaq 'de lo que existe'.

En este sentido, la palabra chaka o chakay significa 'puente' o 'puentear', para hacer cruzar algo de un extremo al otro extremo, mientras que chakatay 'entrelazar, interconectar, entrecruzar'; en ambos casos, hechos o acciones de la naturaleza tanto en forma abstracto o concreto. Por esta afirmación como inferencia del estudio y por esta forma de emisión conceptual filológica, al acto realizado

348 Lengua y Sociedad. Revista de Lingüística Teórica y Aplicada 
por los quechua hablantes, lo llamaríamos relación de significado filosófico, que emerge desde la interacción con lo existente, como en la palabra anca 'cadera' y 'puente' que tiene como concepto de dar una conexión de un elemento con otro, en el que la cadera une el armazón del muslo con la pelvis, el hueso redondo se une con la terminación del fémur en el vacío de la zona pélvica, permitiendo así, la movilidad y la estabilidad de las extremidades, inferior y superior. Mientras que chakata duplica y triplica este hecho en actos mucho más complejos y diversos.

Como categoría lingüística, el término chaka no es sustantivo ni verbo, es una idea base para formar verbos chakay, chakatasqa y chakatay; sustantivos chaka o chakana; adverbios chakanasqa 'atar las cuatro patitas entrecruzadamente'. En la que, según los diccionarios coloniales, chaka significa 'unión' como «anca, cadera y puente» (Anónimo, 1586/2014, p. 79). De la misma manera, con sufijo aglutinados, la palabra chaka + -ta según el Anónimo (1586) es 'palo atravesado a manera de cruz', y chakatani o chakata-y es 'poner en el palo o crucificar. Siendo así, el término chakatay sería el proceso de entrecruzamiento en cuatro direcciones o miles de direcciones con cualquier tipo de elementos abstractos o concretos como palos, sogas, alambres, flujo sanguíneo, energía solar, el calor o acciones en abstractas o concretas, entre otros, pudiendo así entrecruzar a personas, animales, cosas abstractas o entidades concretas. Además, referiremos que chaka es el elemento abstracto, concreto y bioquímico que funciona como base para unir puentes como equilibrio de continuidad de vida que enlaza la secuencia del proceso vital de la existencia; mientras que chakana sería el instrumento con el que se realizan y se apoya para realizar esa conexión de las acciones naturales o humanas.

Y, en extensión, diríamos que también sería la amalgamación de elementos del cosmos que hace un entrecruzamiento en el universo para crear la existencia de los seres. Esto a la vez entendida como algo que se duplica y se multiplica como las acciones de entrecruzamientos de los hechos realizados en la misma naturaleza.

Asimismo ocurriría con las acciones naturales, como por ejemplo la acción que realiza la palabra chacara, chakra o chakara 'chacra', anotado por Szemiński en la obra de Huamán Poma (2005, p. 42), citando a Gonzáles Holguin, como «heredad de labor tierra o huertas». Una segunda entrada como un adjetivo, chacara camayoc «responsable de las tierras cultivadas, el que vive de sementeras, el labrador, el mayordomo de chacras». Entonces, podemos entender la chacra como una zona donde se deposita la semilla, tierra de cultivo, entendido como punto A; el terreno óptimo para el desarrollo de las plantas que hace germinar 
Análisis semántico y hermenéutico de los términos chaka, chakana y chakata Claudia Cisneros Ayala

a las semillas en plantas por influencia de varios factores y luego estás busca los rayos solares para producir el ciclo de vida, esto entendido como punto B. Por la forma de realizar el acto de unir un elemento, la planta, desde el subsuelo y el espacio aéreo, por lo que, la chacra hace cruzar a los vegetales desde el subsuelo hacia el espacio, por ello, los andinos habrían consignado el nombre de chakara o chakra. Y la cruz que se coloca en las tumbas de los difuntos para simbolizar la muerte de alguien, entendida a partir de esta explicación, también filosóficamente sería el traspaso que realiza un estado de vida a otro estado sin vida. Sólo así cobra gran valor el significado de esta palabra, no como cruz con idea de dar muerte a alguien.

Para referir al contexto de la objetividad del estudio chaka sería el calor del sol que se proyecta desde la esencia del mismo Sol hasta la tierra, para originar vida a través de la producción de la fotosíntesis en las plantas. Otro ejemplo objetivo de chakatasqa es el caso del cordero y el chancho al palo, acción que se realiza para asarlos, en donde se atan las patas estirando en palos en cuatro direcciones, formando una «X».

Entonces, si tomamos el significado de entrecruzar como codificación de las características particulares de los hechos de la naturaleza donde se unen dos o más elementos como proceso de unidades biofísico, químico abstractos o concretos, se estaríamos hablando de la interconexión de todo lo que existió y existe en la naturaleza; por ello, se habría rendido la magnitud de su devoción y respeto. Esta sería una explicación simple de la importancia del término chaka y chakatay que el hombre andino haya codificado en su lenguaje como copia de la forma concreta del proceso de la interacción con lo real.

Entendido de esta manera, las leyes naturales no producen cambios en tiempos cortos, es así que los lenguajes que los codifican tampoco producirían cambios en pocos tiempos. El árbol sigue siendo árbol a través del tiempo y en el idioma que fuere, solo cambia esta envoltura para hacer visible en el idioma en el medio donde se desarrolla. La lengua quechua «es una lengua que valora mucho a la naturaleza, ya que es considerado y valorativo con los seres vivos [la tierra, el cielo, el agua, el sol, etc.]» («10 bondades de l a lengua quechua», 2020); y, a partir de ello, se puede conceptualizar los procesos como puentes de amalgamación, tejidos o vínculos de entidades no aisladas que interactúan para producir vida.

Por ello, el estudio se realizó desde distintos enfoques multidisciplinarios y fundamentalmente estudiando la estructura interna del propio idioma. Todo cuanto existe como base en el universo sería chaka y chakatay porque todos 
tienen que hacer puente e interconectar. Nada está aislado, todo se entrecruza en un mismo lugar, animales, personas; los elementos de la naturaleza, el aire, el Sol, la Luna y la Tierra. Y nuestros ancestros habrían plasmado como vínculo vital de lo que existe en la pachamama como representaciones de los símbolos andinos y en el idioma. Tenemos que volver a pensar en una unidad con el cosmos y la naturaleza porque dependemos de ellos para subsistir como seres humanos.

\section{Conclusiones}

Los casos presentados en el análisis de los términos chaka, chakana, chakata y chakatasqa muestran una idea de cómo fue y es el pensamiento filosófico de la cultura andina y la organización de la estructura morfológica de la lengua quechua hace visible y corroborable ese pensamiento como una unidad con los hechos abstractos de lo circundante y también a las acciones humanas.

El idioma quechua cifra la interrelación de los hechos de las leyes de la naturaleza como fenómenos concretos y constantes que generan vida y el idioma quechua solo copia esa realidad.

La categoría léxica del idioma quechua al unirse con sufijos determinados puede formar verbos, como en la palabra chakata con significado 'entrecruz-' solo como lexema; en donde el sufijo - $t a$ significa 'entre' o 'inter'.

El sufijo - $t a$ tiene una función adicional a lo que ya hemos estudiado. Al unirse con base chaka 'cruce', esta palabra chakata se transforma en 'interconect-' de los elementos bio físico, químico, abstracto, natural y humano como acto de pensamiento abstracto.

En el pensamiento andino a través de la lógica del funcionamiento natural estaría dado como aquel objeto que entrecruza «X» entes dentro de la naturaleza, como las estrellas del Cruz del Sur que, por su función astronómica de equinoccio de otoño y primavera, hace que el día y la noche tengan la misma duración, y que, al observar el hecho, a simple vista tiene la forma de «T» o cruz invertida, razón por la cual se habría originado su nombre y además por unir dos puntos opuestos: polo Sur con el polo Norte.

Finalmente, los estudios en el idioma quechua pueden dar luces sobre la explicación y formación de las palabras con su equivalente hispano a través de los campos semánticos al que están interconectados, los elementos de la naturaleza como conceptos y como estructura. 
Análisis semántico y hermenéutico de los términos chaka, chakana y chakata Claudia Cisneros Ayala

Agradezco al Dr. Rufino Gonzalo Espino Relucé mi gran aprecio y gratitud, porque va guiando mis pasos, sugiriendo bibliografías para el desarrollo del material. A mi maestro, Genaro Quispe Pineda, por las sugerencias y críticas que hacen que avance en el campo de la producción y al Dr. Mauro Mamani Macedo, quien orienta mis pasos en la estructura de los escritos. Mi gratitud y estima al Dr. Marco Antonio Lovón Cueva, por guiarme en el desarrollo del presente escrito. Con sus anotaciones y sugerencias atinadas voy incursionando en el terreno de la producción de artículos.

\section{Referencias bibliográficas}

Adelaar, W. (2020). Reconstruction beyond proto-languages in the middle Andes. Cuadernos de Lingüística, 1(1), 1-13. https://cadernos.abralin.org/index.php/ cadernos/article/view/274

Amorín, P. (2007). Gramática del Quechua Ayacuchano. Castilla.

Anónimo. (1856). Vocabulario y phraxis de la lengua General, de los indios del Peru, llamada quichua. Impreso por Antonio Ricardo.

Anónimo. (2014). Arte y vocabulario en la lengua general del Perú. Pontificia Universidad Católica del Perú; Instituto Riva-Agüero. (Obra original publicada en 1586)

Bertonio, L. (2011). Vocabulario de la lengua aymara. Instituto de las Lenguas y Literaturas. (Obra original publicada en 1612)

Carpio N. V. del. (2008). Gestión Pública Intercultural el bien común. Institución Nacional de la Administración Pública República Argentina.

Cerrón-Palomino, R. (2008). Quechumara. Estructuras paralelas del quechua y del aymara. Universidad Mayor de San Simón.

Ciucci, L. y Muysken. (2011). Hernando de Alcocer y la Breve declaración del Arte y Bocabulario de la lengua del Ynga conforme al estilo y vso de la provincia de Quito. El más antiguo manuscrito del quichua Ecuador. Indiana, 28, 359-393. https://www.redalyc.org/pdf/2470/247022796016.pdf

deChile.net. (s. f. a). Codificar. En Diccionario Etimológico Castellano en Línea. Recuperado el 12 de febrero de 2021, de http://etimologias.dechile.net/?codificar deChile.net. (s. f. b). Cruz. En Diccionario Etimológico Castellano en Línea. Recuperado el 12 de febrero de 2021, de http://etimologias.dechile.net/?cruz 
deChile.net. (s. f. c). Entre. En Diccionario Etimológico Castellano en Línea. Recuperado el 12 de febrero de 2021, de http://etimologias.dechile.net/?entre EcuRed. (s. f.). Cruz del Sur (constelación). En EcuRed. Recuperado el 12 de febrero de 2021, de https://www.ecured.cu/Cruz_del_Sur_(constelaci\%C3\%B3n)

García, J. M. (2014). Método filológico, ¿una nueva herramienta? Retos, nuevas tendencias en Educación Física, Deporte y Recreación, 25, 113-116. https:// dialnet.unirioja.es/servlet/articulo? codigo $=4555053$

González Holguin, D. (1989). Vocabulario de la Lengua General de todo el Perú llamada Lengua Qquichua o del Inca. Universidad Nacional Mayor de San Marcos. (Obra original publicada en 1608)

Huamán Poma de Ayala, F. (2005). Nueva Corónica y Buen Gobierno (Tomo III). Fondo de Cultura Económica.

Krogel, A. (2021). Musuq Illa. Poética del Harawi en Runasimi (2000-2020). Pakarina.

Moreno, E. D. (2012). Análisis de las Manifestaciones Culturales que caracterizan a los cuatro Raymicunas en el Cantón Otavalo y Cotacachi en la provincia de Imbabura [Tesis de licenciatura, Universidad Técnica del Norte]. Repositorio Institucional de la Universidad Técnica del Norte. http://repositorio.utn.edu. ec/bitstream/123456789/1630/1/FECYT\%201485\%20TESIS.pdf

Oré, L. J. de. (1598). Symbolo catholico Indiano, en el qual se declaraba los my囚terios dela Fe contenidos enlos tres símbolos Catholicos, Apoftolico, Niceno, y de S. Athanafio. Contiene assi mesmo vna descripción del nuevo orbe, y delos naturalesdel. Y vn orden de enseñarles la doctrina Christiana enlas dos lenguas Generales, Quichua y Aymara, con un Cosfessionario breue y Cateshifmo dela communion. Impreso en Lima por Antonio Ricardo.

Parker, G. J. (1972). Del estado actual de los estudios quechuas. Bulletin de l'Institut Français d'Études Andines, 1(1), 40-51. http://www.ifea.org.pe/libreria/ bulletin/1972/pdf/40.pdf

Pino, A. M. (2012). La chakana ¿Elucubración o resignación? Pluralidades, 1(1), 41-57. https://docplayer.es/49990458-La-chacana-elucubracion-o-resignificacion-ana-maria-pino-jordan.html

Quevedo, M. A. (2012). Análisis de contenido del programa La Chakana y sus mensajes de interculturalidad en la radio CCE [Trabajo de grado, Universidad Central del Ecuador]. Repositorio Digital de la Universidad Central del Ecuador. http://www.dspace.uce.edu.ec/bitstream/25000/757/1/T-UCE-0009-15.pdf 
Ricardo, A. (Impresor). (1584). Doctrina christiana y catecismos de los indios, y

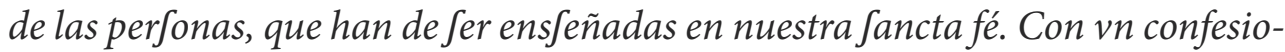
nario, y otras cosas neceffarias para los que doctrinan. Traduzido en dos lenguas generales, de este reyno, quichua, y ayma. Impreso por Antonio Ricardo.

Santo Tomás, D. de (1951). Lexicón o vocabulario de la lengua general del Perú. Instituto de Historia de la Universidad Nacional Mayor de San Marcos. (Trabajo original publicado en 1560)

Szabó, H. E. (2008). Chakana. En Diccionario de la antropología boliviana (p. 191). Aguaragüe.

Timmer, H. (. (2003). La chakana [Documento en internet]. Alquimia Abdominal. https://www.alquimiaabdominal.cl/wp-alquiminal/uploads/2015/12/La_ Chakana.pdf

Torre, C. de la. (2002). Chakana: Meditación y oración en la cultura andina. ITDG-Perú.

Torres Rubio, D. de. (1619). Arte de la Lengua Qvichua. Impreso por Francisco Lasso.

Urton, G. (1983). El sistema de orientaciones de los incas y de algunos quechuahablantes actuales tal como queda reflejado en su concepto de la astronomía y del universo. Anthropologica, 1(1), 209-238. https://revistas.pucp.edu.pe/index. php/anthropologica/article/view/6311

Yampara H., S. e. (2005). Comprensión aymara de la Tierra-territorio en la cosmovisión andina y su ordenamiento para la/el qamaña. Revista Inti-Pacha, 1-7, 13-44. https://studylib.es/doc/7446849/cosmovisi\%C3\%B3n-territorial-ecolog\% $\mathrm{C}_{3} \%$ ADa-y-medio-ambiente

Zúñiga Tinizaray, V. A. (2006). Aproximación a un Vocabulario Visual Básico Andino [Tesis de maestría, Universidad de Palermo]. Facultad de Diseño y Comunicación de la Universidad de Palermo. https://www.palermo.edu/dyc/ maestria_diseno/pdf/tesis.completas/05\%20Zuniga.pdf

10 bondades de la lengua quechua. (2020, 28 de mayo). T\&T Cecitel. http://tytcecitel.edu.pe/con-estrateg/10-bondades-de-la-lengua-quechua/ 


\section{Trayectoria académica de la autora}

Claudia Cisneros Ayala es licenciada en Lengua y Literatura, con una Segunda Especialidad de Educación Rural Intercultural Bilingüe. Tiene una maestría en Didáctica de la Comunicación por la Universidad Nacional de Educación, Enrique Guzmán y Valle. Es traductora e intérprete de lenguas indígenas del Ministerio de Cultura y con certificación de Competencias del Experto en Comunicación en Lenguas Indígenas u Originarias de SINEACE. Actualmente, es candidata a doctorado en Lingüística. Es especialista en quechua, docente de literatura AndinaAmazónica. Sus intereses se centran en el estudio, difusión de la cultura andina y enseñanza del idioma quechua con identidad cultural. 